\title{
EARLY DETECTION OF DROUGHT STRESS IN ARABIDOPSIS THALIANA UTILSING A PORTABLE HYPERSPECTRAL IMAGING SETUP
}

\author{
Puneet Mishra $^{1 *}$,Torsten Feller ${ }^{2 *}$, Martin Schmuck ${ }^{2}$, Andreas Nicol ${ }^{2}$, Alison Nordon ${ }^{1 *}$ \\ ${ }^{1}$ WestCHEM, Department of Pure and Applied Chemistry and Centre for Process Analytics and Control \\ Technology, University of Strathclyde, Glasgow, G1 1XL, United Kingdom \\ ${ }^{2}$ Bayer AG, Engineering \& Technology, Screening Technology, Building E 41, 51368 Leverkusen, \\ Germany
}

\begin{abstract}
Close-range hyperspectral imaging (HSI) of plants is now a potential tool for non-destructive extraction of plant functional traits. A major motivation is the plant phenotyping related applications where different plant genotypes are explored for different environmental conditions. HSI of Arabidopsis thaliana is of particular importance as it is a model organism in plant biology. In the present work, a portable HSI setup has been used for the monitoring of a set of 6 Arabidopsis thaliana plants. The plants were monitored under controlled watering conditions where 3 plants were watered as normal and the other 3 plants were given $50 \%$ of the normal volume of water. The images were pre-processed utilising the standard normal variate (SNV) and changes over time were evaluated using unsupervised clustering over the time series. The results showed an early detection of stress from day 4 onwards compared to the commonly used normalised difference vegetation index (NDVI), which provided detection from day 9.
\end{abstract} Index Terms - visible-near infrared, phenotyping, digital,
agriculture, automatic

\section{INTRODUCTION}

Imaging technologies are emerging as potential tools for the rapid non-destructive understanding of the functional traits of plants [1]. Further, HSI in particular, is becoming a key tool for studying the functional dynamics of plants. A reason for this is that HSI combines two complementary modalities i.e. imaging and spectroscopy. The imaging modality provides access to the structure of the plants whereas the spectroscopy provides insight into the chemical state of the plants. Visible and near-infrared spectroscopy (VNIRS (400-1000nm)) HSI is of particular interest as it provides access to the key chemical pigments, which can be directly relatable to the photosynthetic activity of plants as well as the moisture and internal structure of plant leaves [2].
There is significant interest in of utilising close range HSI for phenotyping applications where the growth of the plants belonging to a particular genotype is monitored with respect to its surrounding environment [3]. Monitoring the plant in such a way allows the physicochemical changes in the plants to be followed and quantified, assisting with the search for a target gene. In plant biology, Arabidopsis thaliana has emerged as the model plant to support such testing experiments [4]. There are different benefits associated with studying Arabidopsis thaliana as it develops, reproduces and responds to stress and diseases in much the same way as many crop plants. The knowledge developed on the Arabidopsis thaliana can be applied directly to other crop plants. Further, it is an easy and inexpensive plant with a rapid life cycle involving prolific seed production thus supporting large scale experiments involving thousands of plants [5].

Laboratory-scale phenotyping and plant biology experiments are usually performed in controlled environmental conditions such as greenhouses. In recent years, to automate the handling of large-scale experiments and measurements, the high-throughput plant phenotyping setups (HTPPS) have emerged [6]. HSI can also be integrated into HTPPS, which support the continuous monitoring of the plants. However, when HSI is integrated within HTPPS, its use is limited to large scale experiments and it cannot be used as a portable tool to explore the plants in small scale settings. Hence, this study aims to explore the potential of a portable HSI setup, which can be used with a wider range of experimental setups and is not restricted to HTPPS.

The objective of this study is to utilise a portable HSI setup for imaging the stress responses in Arabidopsis thaliana. The study involved monitoring a set of Arabidopsis thaliana plants, where half of the plants were normally watered and the other half were given only $50 \%$ of the required water. The HS images were processed with unsupervised clustering to detect the stress symptoms as early as possible. The results were also compared with the standard NDVI to prove the potential of HSI over multispectral cameras. 


\section{MATERIAL AND METHODS}

\subsection{Image acquisition}

Images were recorded with an automatic portable HSI setup developed recently in the Screening Technology Department at Bayer AG. To record the images the setup utilises a push broom line-scan hyperspectral camera from Headwall Photonics, Massachusetts, USA. The camera recorded the image with a spectral range of $400-1000 \mathrm{~nm}$ recording 270 spectral bands with 640 spatial pixels. The number of spatial pixels recorded in each scan was $1004 \times 1004$. The illumination was provided with two halogen bulbs aligned at $45^{\circ}$ to the field of view of the camera. Imaging of the plant was performed by keeping the plant still at the field of view (FOV) and moving the camera using a motorised stage to record the plant and the white reference standard (Spectralon).

\subsection{Samples description}

The study involved monitoring 6 different Arabidopsis thaliana plants. Out of the 6 , half of the plants were well watered and the other half were given $50 \%$ of the required water. The study was performed in an indoor laboratory environment and all the samples were stored under similar conditions. The samples were monitored for 10 days over the period from $23^{\text {rd }}$ July 2018 to $3^{\text {rd }}$ August 2018.

\subsection{Data processing}

\subsubsection{Radiometric calibration}

Variations in signal arising from illumination intensity, the detector sensitivity and the transmission properties of the optics were corrected by radiometric calibration utilising dark and white reference images. The correction was performed for every pixel in the hyperspectra (HS) image according to equation (1):

$$
I_{R}(i, j, k)=\frac{I_{\text {raw }}(i, j, k)-I_{\text {dark }}(i, j, k)}{I_{\text {white }}(i, j, k)-I_{\text {dark }}(i, j, k)}
$$

where, $I_{R}$ is the calibrated reflectance, $I_{\text {raw }}$ is the raw intensity measured from the test sample, $I_{\text {dark }}$ is the intensity of the dark response, $I_{\text {white }}$ is the intensity of the uniform white reference, and $i$ and $j$ are spatial coordinates and $k$ is the wavelength of the image.

\subsubsection{Normalisation and smoothing}

The spectral range of the hypercube was reduced to $450-$ $900 \mathrm{~nm}$ to remove the noisy part of the spectrum. Further, a moving window Savitzky-Golay filter [7] (15-point width and second order polynomial) was applied to each pixel of the image to remove random noise, e.g. spikes, from spectra. Further, to reduce light scattering effects arising from inhomogeneity of the leaf surface, the spectra were normalised using the standard normal variate (SNV) transformation $[6,8,9]$.

\subsubsection{NDVI estimation}

NDVI was estimated for each image to benchmark the unsupervised methodology presented in this work. To represent an image a singles NDVI value was estimated as an average of the all NDVI values calculated for each pixel of the complete plant. The NDVI for the HS image of plants can be calculated using equation (2):

$$
N D V I(i, j)=\frac{I_{R}(i, j, 740)-I_{R}(i, j, 670)}{I_{R}(i, j, 740)+I_{R}(i, j, 670)}
$$

where $i$ and $j$ are spatial coordinates and the values of 670 and 740 are wavelengths (in $\mathrm{nm}$ ).

\subsubsection{Clustering}

To extract the meaningful information from the HS image, the $k$-means clustering algorithm was used (without initial seeds), where the appropriate number of clusters $k$ was estimated using the elbow method. The elbow method looks at the percentage of variance explained as a function of the number of clusters. The number of clusters chosen explained $90 \%$ of the variance in the data. Clustering was performed on spectra that were extracted from a subset of images. The subset comprised two images, one from a drought plant and the other from a well-watered plant that was monitored each day over the 10 day time period. Based on the cluster centroids extracted the clusters were assigned to all other plants time series images utilising Euclidean distance. The cluster maps were later reshaped to the image size and the cluster proportions were then plotted and visualised. The cluster proportions were estimated by taking the ratio of pixels belonging to a particular cluster to that of the total number of pixels corresponding to the plant.

\section{RESULTS}

Figure 1 presents the elbow plot for the clustering performed on the spectra of plants acquired over the 10 day time period. The number of clusters was selected based on a threshold of $90 \%$ variance, which resulted in the selection of 8 clusters for the k-means analysis.

Figure 2 presents the cluster centroids obtained from the clustering. It can be noted that the scale of cluster centroids is from -2 to 1.5 and not 0 to 1 as for typical reflectance measurement, a reason for this is that the clustering was performed on the normalised spectra. In previous work, 
spectral normalisation was shown to support the clustering of plant data by reducing the effects of variation in illumination conditions in images [6,9]. Figure 2 shows that the cluster centroids have variation over the complete spectral range however at particular regions such as $550 \mathrm{~nm}$ and $670 \mathrm{~nm}$ there is larger variation.

A reason for these differences could be understood as the change in the concentration of photosynthetic pigments i.e. chlorophyll and anthocyanin. The spectral bands around 550 $\mathrm{nm}$ and $670 \mathrm{~nm}$ arise from the anthocyanin and chlorophyll pigments and the concentrations of these pigments are affected by any stress that the plant may be suffering. In a typical response to stress, the chlorophyll pigments decrease and the anthocyanin increases, which results in the plant looking less green and more red or brown.

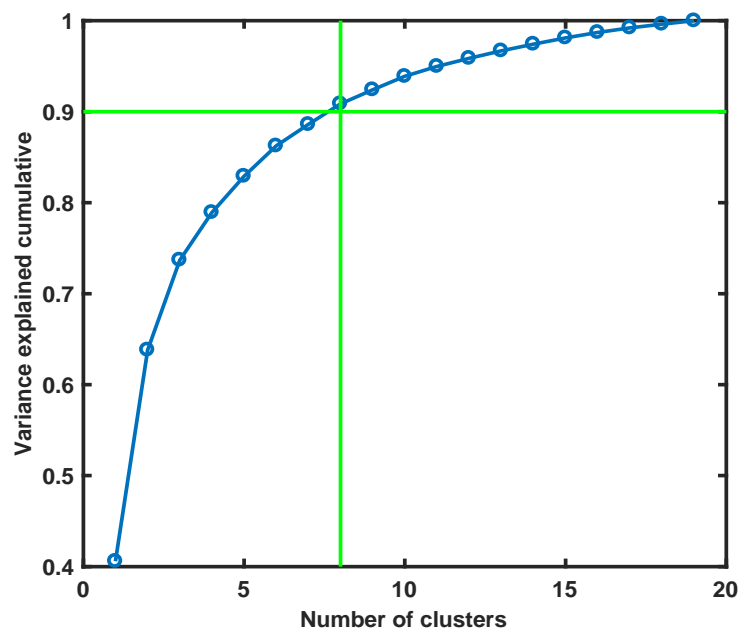

Figure 1: Criterion for selection of the number of clusters to explain at least $90 \%$ of the variance in the data.

Figure 3 presents the reconstructed cluster maps obtained from the k-means clustering. The cluster maps from the last five days of the measurement are provided. It can be seen in Figure 3 that the plants that were given $50 \%$ less water started to show the evolution of new clusters whereas the number of clusters in the normally watered plants remained constant. The number of pixels belonging to the new clusters increases with time owing to drought stress. The cluster proportions were quantified and the results for cluster 2 are presented in Figure 4.

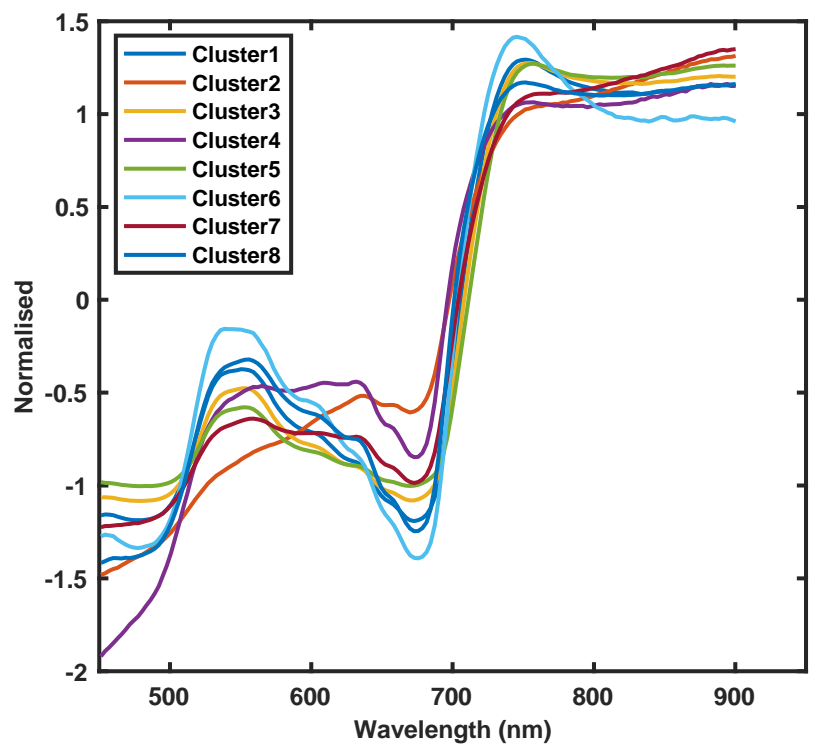

Figure 2: 8 cluster centroids from K-means.

Cluster 2 was chosen as it was found to provide the earliest clear separation of the well-watered and the plant under drought stress. Figure 4 (a) presents the average NDVI values along with the standard deviation over the complete time period of the experiment. Figure 4(b), provides the evolution of the proportion of pixels belonging to cluster 2 along with the standard deviation for the complete time series. In Figure 4(a) it can be noted that with NDVI a clear separation was only possible on day 9 after the stress induction whereas with the clustering approach it can be seen that the plant can be separated after the $4^{\text {th }}$ day of stress induction, thus demonstrating the benefit of HSI for studying Arabidopsis thaliana plant.

\section{CONCLUSIONS}

The present work showed that HSI can be a potential tool to image the functional dynamics of Arabidopsis thaliana. The results showed that HSI provided early detection of drought stress compared to the standard NDVI. The clustering approach showed that an unsupervised data processing algorithm can be used to identify and localise the stressrelated changes in the plant. The changes can further be quantified and viewed as evolution plots to make the judgment on early stress detection. In future work, the methodology will be tested on different stress conditions for Arabidopsis thaliana. 

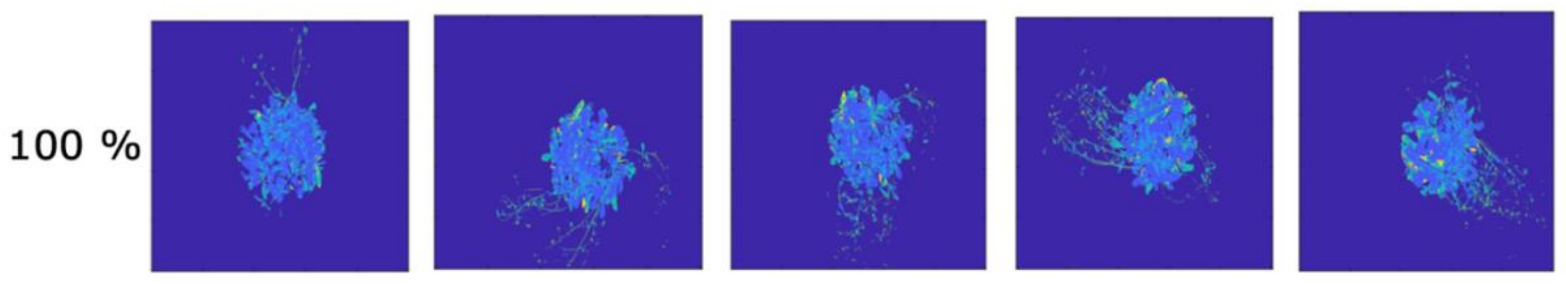

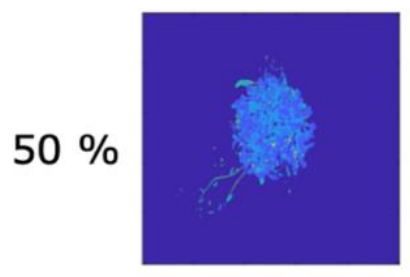

30-07-2018

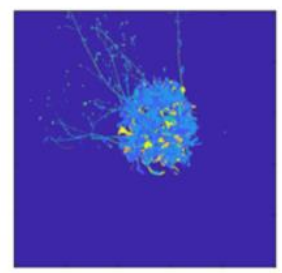

31-07-2018

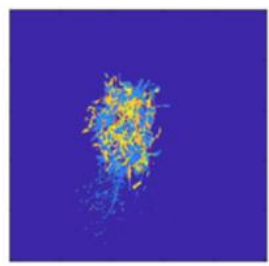

01-08-2018

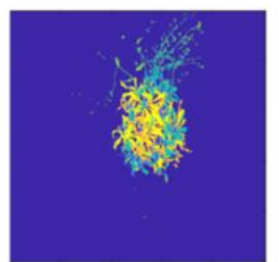

02-08-2018

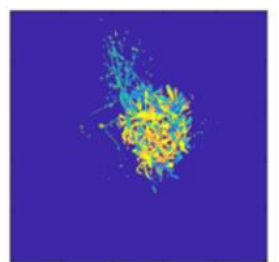

03-08-2018

Figure 3: Evolution of clusters in well-watered (100\%) plants and plants given $50 \%$ of the normal volume of water.

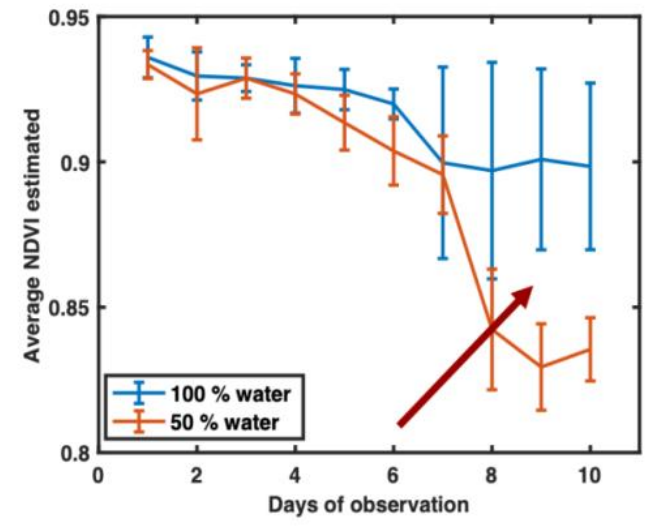

(a). NDVI

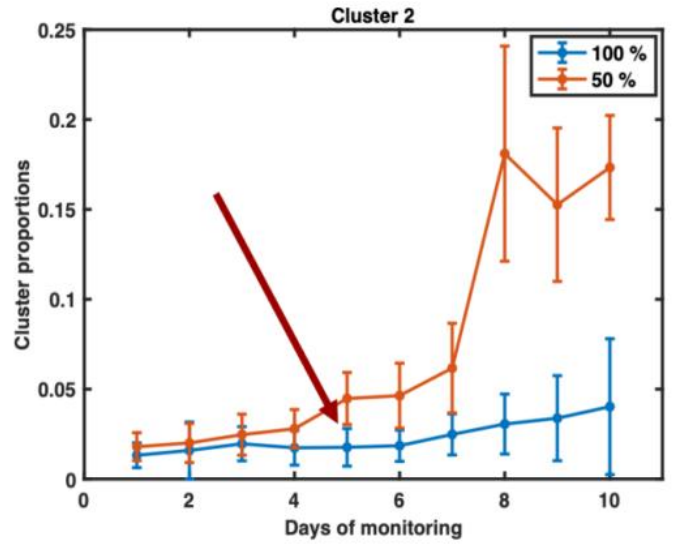

(b). Clustering

Figure 4: Evolution of (a) NDVI and (b) the proportion of cluster 2 during the 10 days of measurements.

\section{ACKNOWLEDGMENTS}

This work has received funding from the European Union's Horizon 2020 research and innovation program under the Marie Skłodowska-Curie grant agreement number 675251

\section{REFERENCES}

[1] N. Fahlgren, M.A. Gehan, I. Baxter, "Lights, camera, action: high-throughput plant phenotyping is ready for a close-up," Current Opinion Plant Biology, 24 (2015) 93-99.
[2] P. Mishra, M.S.M. Asaari, A. Herrero-Langreo, S. Lohumi, B. Diezma, P. Scheunders, "Close range hyperspectral imaging of plants: A review,” Biosystem Engineering, 164 (2017) 49-67.

[3] L. Li, Q. Zhang, D. Huang, “A Review of Imaging Techniques for Plant Phenotyping," Sensors, 14 (2014) 20078-20111.

[4] S. Gepstein, B.A. Horwitz, "The impact of Arabidopsis research on plant biotechnology," Biotechnology Advances, 13 (1995) 403-414. 
[5] M. Bevan, S. Walsh, "The Arabidopsis genome: a foundation for plant research," Genome Research, 15 (2005) 1632-1642.

[6] M.S. Mohd Asaari, P. Mishra, S. Mertens, S. Dhondt, D. Inzé, N. Wuyts, P. Scheunders, "Close-range hyperspectral image analysis for the early detection of stress responses in individual plants in a high-throughput phenotyping platform," ISPRS Journal Photogrammetry Remote Sensing, 138 (2018) 121-138.

[7] A. Savitzky, M.J.E. Golay, "Smoothing and Differentiation of Data by Simplified Least Squares Procedures," Analytical Chemistry, 36 (1964) 1627-1639.

[8] R.J. Barnes, M.S. Dhanoa, S.J. Lister, "Standard Normal Variate Transformation and De-Trending of Near-Infrared Diffuse Reflectance Spectra," Applied Spectroscopy, 43 (1989) 772-777.

[9] M.A.M. Shahrimie, P. Mishra, S. Mertens, S. Dhondt, N. Wuyts, P. Scheunders, "Modeling effects of illumination and plant geometry on leaf reflectance spectra in close-range hyperspectral imaging," in: 2016 8th Work. Hyperspectral Image Signal Process. Evol. Remote Sens., 2016: pp. 1-4. 